\title{
Las intervenciones no farmacológicas podrian reducir los síntomas depresivos en los adultos mayores
}

Non-pharmacological interventions may reduce depressive symptoms in older adults

\section{Objetivos}

Valorar la efectividad de los tratamientos no farmacológicos para la depresión en adultos mayores.

\section{Fuentes de datos}

Búsqueda inicial en MEDLINE y CINAHL, seguida de una búsqueda de estudios publicados y no publicados entre 2000 a 2012 en una amplia variedad de bases de datos de ciencias de la salud.

\section{Selección de estudios y extracción de datos}

Se incluyeron artículos publicados en inglés, español y portugués
Apóstolo J, y col. Int J Nurs Stud. 2016;58:59-70. que incluyeran adultos mayores de 65 años con cualquier tipo de trastorno depresivo, sin tener en cuenta las comorbilidades y tratamientos previos, excluyendo a las personas con episodios maníacos o síntomas psicóticos.

\section{Resultados Principales}

Debido a que los estudios incluidos valoraron intervenciones no farmacológicas técnicamente disímiles, la agrupación de datos para elaborar un meta análisis fue posible solamente para dos estudios (Gellis y Bruce 2010, y Gellis y col. 2008). En la tabla 1 se describen los principales resultados.

Tabla 1. Características de los estudios incluidos y medidas de efecto de sus intervenciones no farmacológicas para reducir los síntomas depresivos en adultos mayores.

\begin{tabular}{|c|c|c|c|c|}
\hline Resultado & Diseño & n & Intervención y comparación & Medidas de efecto (e instrumento) \\
\hline $\begin{array}{l}\text { Serfaty y col. (2009) } \\
\text { Reino Unido }\end{array}$ & ECCA & 204 & $\begin{array}{l}\text { TCC vs tratamiento usuala } \\
\text { TCC vs tratamiento usual }+ \text { charlas control }^{a}\end{array}$ & $\begin{array}{l}\text { Diferencia de medias -3,07 (efecto pequeño); IC95\% -5,73 a -0,42 } \\
\text { para Inventario de depresión de Beck-II } \\
\text { Diferencia de medias: -3,65 (efecto pequeño); IC95\% -6,18 a -1,12 } \\
\text { para Inventario de depresión de Beck-II } \\
\text { Se evaluó también calidad de vida para la cual no se encontraron } \\
\text { diferencias. }\end{array}$ \\
\hline $\begin{array}{l}\text { Ekkers y col.(2011) } \\
\text { Países Bajos }\end{array}$ & ECCA & 93 & $\begin{array}{l}\text { Entrenamiento competitivo de la memoria } \beta \text { vs } \\
\text { tratamiento usual } \beta \text { }\end{array}$ & $\begin{array}{l}\text { Menores síntomas depresivos: } p=0,01 \text {; Cohen's } d=0,54 \text { (efecto } \\
\text { moderado) para Geriatric Depression Scale- } 30 \text { y } p<0,000 \text {; Cohen's } \\
d=0,66 \text { (efecto moderado) para Quick Inventory of Depressive } \\
\text { Symptomatology-Self Report; } p=0,005 \text {; Cohen's } d=0,58 \text { (efecto } \\
\text { moderado) para Sadness Scale }\end{array}$ \\
\hline $\begin{array}{l}\text { Su y col (2012) } \\
\text { Taiwan }\end{array}$ & $\begin{array}{l}\text { Cuasi-experimental } \\
\text { (antes después) }\end{array}$ & 93 & $\begin{array}{l}\text { Terapia de grupo de reminiscencia }{ }^{\complement} \text { vs } \\
\text { entrevistas de apoyo uno a uno. }\end{array}$ & $\begin{array}{l}\text { Menores síntomas depresivos en el grupo intervención grupal ( } p= \\
0.02 \text {, magnitud no disponible); sin embargo, no se observó un efecto } \\
\text { significativo con la terapia individual }\end{array}$ \\
\hline $\begin{array}{l}\text { Kiosses y col. (2010) } \\
\text { EE.UU. }\end{array}$ & ECCA & 30 & $\begin{array}{l}\text { Terapia de adaptación al problema }{ }^{*} \text { vs } \\
\text { intervención domiciliaria inespecífica* }\end{array}$ & $\begin{array}{l}\text { Menores síntomas depresivos en el grupo intervención } p=0,0307 \\
\text { (magnitud no disponible) }\end{array}$ \\
\hline $\begin{array}{l}\text { Gellis y Bruce (2010) } \\
\text { EE.UU. }\end{array}$ & ECCA & 38 & $\begin{array}{l}\text { Terapia de resolución de problemas }{ }^{\epsilon} \text { VS } \\
\text { cuidado usual + educación }\end{array}$ & $\begin{array}{l}\text { Menores síntomas depresivos ( } p=0,008 \text { para el Inventario de } \\
\text { depresión de Beck; } p=0,006 \text { para el Hamilton Depression Rating } \\
\text { Scale-17; magnitud no disponible) }\end{array}$ \\
\hline $\begin{array}{l}\text { Gellis y col. (2008) } \\
\text { EE.UU. }\end{array}$ & ECCA & 62 & & $\begin{array}{l}\text { Menores síntomas depresivos ( } p<0,001 \text { para el Hamilton Depression } \\
\text { Rating Scale-17; } p<0,001 \text { para el Geriatric Depression Scale-15, } \\
\text { magnitud no disponible) } \\
\text { Sin diferencias en la calidad de vida }\end{array}$ \\
\hline
\end{tabular}

TCC: terapia cognitivo conductual. ECCA: ensayo clínico controlado y aleatorizado. EE.UU.: Estados Unidos de Norte América. RRR: reducción de riesgo relativo.

aCharlas sobre temas neutrales como pasatiempos, deportes, actualidad (no se aconsejaba sobre resolución de problemas ni se trataban temas emocionales).

aaMedicación, cuidados habituales, derivación a otros servicios.

B Se enfoca fundamentalmente en estrategias de manejo de rumiación de ideas.

B B Tratamiento farmacológico +/- psicoterapia.

£ Terapia grupal administrada en 72 sesiones de dos horas enfocada en temas varios: "conocerse unos a otros", "niñez y familia", "empleos previos para ganarse la vida", etc.

$¥$ Intervención domiciliaria ("en el ecosistema del paciente") enfocada a la resolución de problemas y adaptación funcional.

$¥ ¥$ Intervención domiciliaria inespecífica: escucha empática, reflexión, aliento, etc.

$€$ Sesiones domiciliarias administradas en seis sesiones de una hora enfocadas en el desarrollo de habilidades específicas de resolución o afrontamiento de problemas.

\section{Conclusiones}

Las intervenciones no farmacológicas tuvieron efectos positivos en la mejora de los síntomas de la depresión en los pacientes. Sin embargo, debido a la diversidad de intervenciones y al bajo número de estudios por intervención incluidos en esta revisión sistemática, la evidencia no es lo suficientemente fuerte para producir una guía de recomendación de buenas prácticas.

Fuente de financiamiento/conflicto de interés de los autores: no referida. 


\section{Comentario}

La depresión afecta a un $7 \%$ de la población de los adultos mayores en general. En los establecimientos de atención primaria de salud la depresión tiende a subdiagnosticarse y en ocasiones subtratarse ya que los síntomas coinciden con otros problemas que experimentan los adultos mayores. En algunos casos, la disminución y/o pérdida de la independencia por dificultades en la movilidad, dolor crónico, fragilidad, problemas mentales o físicos, maltrato, fallecimiento de un ser querido, jubilación y discapacidad, puede ocasionar aislamiento, pérdida de la independencia, soledad, angustia y percepción de mala salud con el consecuente aumento en la utilización de los servicios médicos y los costos de la asistencia sanitaria ${ }^{1}$. Esta revisión, si bien mostró que las intervenciones evaluadas pueden reducir significativamente los puntajes de síntomas depresivos, no pudo demostrar efectos beneficiosos de estas sobre la calidad de vida o el funcionamiento cognitivo. Debido a la diversidad de intervenciones y al bajo número de estudios por intervención incluidos en esta revisión sistemática, no pueden extraerse conclusiones lo suficientemente fuertes como para establecer una recomen- dación clínica. Por otra parte, existen pocos resultados sobre la efectividad de las intervenciones no farmacológicas considerando la presencia o ausencia de tratamiento farmacológico simultáneo para la depresión, presencia de comorbilidades y gravedad de los síntomas. Esta limitación también ha sido mencionada por los autores de los estudios incluidos.

\section{Conclusiones del comentador}

Es importante abordar el problema de la depresión en la consulta de atención primaria, detectar factores de riesgo como la percepción de soledad, ausencia de red de contención, comorbilidades, y disminución de la funcionalidad; trabajando en los intereses propios, fortalecimiento social y capacidad funcional, manteniendo la visión de atención centrada en la persona. Las medidas no farmacológicas podrían constituir una herramienta útil en el abordaje integral de esta problemática. Sin embargo se requiere evidencia de mejor calidad para poder hacer recomendaciones claras respecto de su indicación formal.

Roxana E. Ramos [ Programa Sociosanitario para el Adulto Mayor Frágil del Hospital Italiano de Buenos Aires. roxana.ramos@ hospitalitaliano.org.ar ]

Ramos RE. Las intervenciones no farmacológicas podrían reducir los síntomas depresivos en los adultos mayores. Evid Act Pract Ambul 2017;20(2):4243. Comentado de: Apóstolo J, y col. The effectiveness of non pharmacological interventions in older adults with depressive disorders: a systematic review. Int J Nurs Stud. 2016 Jun;58:59-70. PMID: 27087298.

Referencias

1. La salud mental y los adultos mayores [en linea]. Organización Mundial de la Salud. Abril 2016. Disponible en URL: http://www.who.int/mediacentre/factsheets/fs381/es/\#. WDy7lism_zs.email (último acceso agosto 2017)

2. Ribot Reyes VC, y col. Suicidio en el adulto mayor. Revista Habanera de Ciencias Médicas 2012;11(5):699-708 